\title{
2-(Trimethylsilyloxy)furan as a dianion equivalent: a two-step synthesis of functionalised spirocyclic butenolides.
}

\author{
Nuno Maulide and István E. Markó* \\ Université catholique de Louvain, Département de Chimie, Bâtiment Lavoisier \\ Place Louis Pasteur 1, 1348 Louvain-la-Neuve, Belgium.
}

\section{SUPPORTING INFORMATION}

\author{
Table of contents
}

Experimental procedures .................... S $1-\mathrm{S} 6$
Spectra for selected intermediates......... S $7-\mathrm{S} 15$

Instrumentation and procedures.

Routine nuclear magnetic resonance (NMR) spectra were recorded on a VARIAN GEMINI200, VXR-200 ( ${ }^{1} \mathrm{H} 200 \mathrm{MHz}$ and $\left.{ }^{13} \mathrm{C} 50 \mathrm{MHz}\right)$, VARIAN GEMINI-300 ( ${ }^{1} \mathrm{H} 300 \mathrm{MHz}$ and $\left.{ }^{13} \mathrm{C} 75 \mathrm{MHz}\right)$, BRUKER-300 $\left({ }^{1} \mathrm{H} 300 \mathrm{MHz}\right.$ and $\left.{ }^{13} \mathrm{C} 75 \mathrm{MHz}\right)$ and BRUKER-500 $\left({ }^{1} \mathrm{H} 500\right.$ $\mathrm{MHz}$ and $\left.{ }^{13} \mathrm{C} 125 \mathrm{MHz}\right)$ spectrometers. Chemical shifts $\left(\delta_{\mathrm{H}}\right)$ are reported in ppm downfield from internal tetramethylsilane (TMS). ${ }^{13} \mathrm{C}$ NMR spectra were recorded using $\mathrm{CDCl}_{3}$ as the internal standard. Splitting patterns in ${ }^{1} \mathrm{H}$ spectra are designed as $\mathrm{s}$, singlet; d, doublet; $\mathrm{t}$, triplet; $b$, broad and $\mathrm{m}$, multiplet.

Low resolution mass spectra (EI) were recorded using VARIAN MATT-44 and FINNIGAN MAT-TSQ 70 spectrometers.

Infra-red (IR) spectra were taken as $\mathrm{KBr}$ disks, thin films or in solution, on a SHIMADZU Benelux FTIR-8400S spectrometer and recorded in $\mathrm{cm}^{-1}$.

Elemental analyses were performed in Prof. S. Laschat's analytical laboratory (Institut für Organische Chemie, Universität Stuttgart, Germany). High-resolution mass spectra were recorded in Prof. R. Flamant's laboratory (Université de Mons, Belgium).

Thin layer chromatography (TLC) was performed on MERCK silica gel $60 \mathrm{~F}_{254}$ aluminumbacked plates. The plates were visualised using $254 \mathrm{~nm}$ UV light and developed using an alkaline $\mathrm{KMnO} 4$ solution $\left(1 \% \mathrm{KMnO}_{4} / 5 \% \mathrm{Na}_{2} \mathrm{CO}_{3}\right)$. Flash chromatography was performed using Rocc silica gel $60(40-63 \mu \mathrm{m})$ under pressure with the stated solvents. 
All solvents were routinely distilled prior to use. Reactions were performed under a dry, inert atmosphere of argon unless stated otherwise.

The following abbreviations are used: rt, room temperature; hr, hour; min, minute; mmol, millimole; mol, mole; mg, milligram; g, gram; mL, milliliter, $\mu \mathrm{L}$, microliter; eq., equivalent. 


\section{General procedure for the preparation of spirocyclic butenolides $7 b-7 i$.}

A solution of the corresponding butenolide ketal $(1 \mathrm{mmol}, 1$ eq.) in $10 \mathrm{~mL}$ THF was cooled to $0{ }^{\circ} \mathrm{C}$ and treated with $1.3 \mathrm{mmol}(1.3$ eq.) of either a $2.0 \mathrm{M}$ THF solution of NaHMDS or solid $t$-BuOK. The reaction was stirred until complete conversion of the starting materials and quenched by addition of a saturated aqueous $\mathrm{NH}_{4} \mathrm{Cl}$ solution. Following extractive workup, purification by flash chromatography on silica gel (elution with 10\% EtOAc/hexane) afforded the desired products.

$7 \mathbf{b}$

${ }^{1} \mathrm{H}$ NMR $\left(300 \mathrm{MHz}, \mathrm{CDCl}_{3}\right): \delta 7.33(\mathrm{~d}, J=5.7 \mathrm{~Hz}, 1 \mathrm{H}), 6.08(\mathrm{~d}, J=5.7 \mathrm{~Hz}, 3 \mathrm{H}), 3.97-3.70$ (m, $4 \mathrm{H}), 2.25-2.13(\mathrm{~m}, 2 \mathrm{H}), 2.01-1.79(\mathrm{~m}, 4 \mathrm{H}) ;{ }^{13} \mathrm{C} \mathrm{NMR}\left(75 \mathrm{MHz}, \mathrm{CDCl}_{3}\right): \delta 154.4,122.8$, 116.9, 95.6, 66.5, 65.1, 33.6, 32.3, 18.3; IR( $\left(\mathrm{cm}^{-1}\right): 3359,2921,1751,1697,1391$; MS (APCI+ev): $m / z(\%): 197(100)[M+H]^{+}, 135(5), 99$ (5); Elemental analysis calcd. for $\mathrm{C}_{10} \mathrm{H}_{12} \mathrm{O}_{4} \mathrm{C}: 61.26 \%$; H $6.17 \%$; found C 60.82\%; H 6.16\%.

$7 \mathrm{c}$

${ }^{1} \mathrm{H}$ NMR $\left(300 \mathrm{MHz}, \mathrm{CDCl}_{3}\right): \delta 7.39(\mathrm{~d}, J=6.0 \mathrm{~Hz}, 1 \mathrm{H}), 6.08(\mathrm{~d}, J=6.0 \mathrm{~Hz}, 1 \mathrm{H}), 4.01-3.77$ (m, $4 \mathrm{H}), 1.90-1.54(\mathrm{~m}, 8 \mathrm{H}) ;{ }^{13} \mathrm{C} \mathrm{NMR}\left(75 \mathrm{MHz}, \mathrm{CDCl}_{3}\right): \delta 155.7,122.4,116.4,94.9,65.9$, 65.8, 33.4, 29.7, 22.8, 21.5; IR( $\left(\mathrm{cm}^{-1}\right): 2968,1751,1143$; MS (APCI+ev): $m / z(\%): 211$ (100) $[M+H]^{+}$, 149 (15), 121 (5); HRMS (ES) calcd. for $\mathrm{C}_{11} \mathrm{H}_{14} \mathrm{O}_{4} \mathrm{Na}(\mathrm{M}+\mathrm{Na})$ : 233.0790; found 233.0795 .

$7 d$

${ }^{1} \mathrm{H}$ NMR $\left(300 \mathrm{MHz}, \mathrm{CDCl}_{3}\right): \delta 6.94(\mathrm{~d}, J=2.1 \mathrm{~Hz}, 1 \mathrm{H}), 4.07-3.73(\mathrm{~m}, 4 \mathrm{H}), 2.23-2.14(\mathrm{~m}, 2$ H), 1.99-1.81 (m, $4 \mathrm{H}), 1.93(\mathrm{~s}, 3 \mathrm{H}) ;{ }^{13} \mathrm{C} \mathrm{NMR}\left(75 \mathrm{MHz}, \mathrm{CDCl}_{3}\right): \delta 172.3,146.6,131.4$, 116.7, 93.2, 66.4, 65.1, 33.5, 32.4, 18.2, 10.9; IR( $\left(\mathrm{cm}^{-1}\right): \operatorname{IR}\left(\mathrm{cm}^{-1}\right): 3360,2919,2844,1759$, 1655, 1415; MS (APCI+ev): $m / z(\%): 211(100)[M+H]^{+}, 149$ (10), 99 (5); Elemental analysis calcd. for $\mathrm{C}_{11} \mathrm{H}_{14} \mathrm{O}_{4} \mathrm{C}: 61.38 \%$; $\mathrm{H} 6.80 \%$; found $\mathrm{C} 61.85 \% ; \mathrm{H} 6.71 \%$.

$7 e$

${ }^{1} \mathrm{H}$ NMR $\left(300 \mathrm{MHz}, \mathrm{CDCl}_{3}\right): \delta 7.00(\mathrm{~d}, J=1.5 \mathrm{~Hz}, 1 \mathrm{H}), 3.97-3.80(\mathrm{~m}, 4 \mathrm{H}), 1.91(\mathrm{~d}, J=1.5$ $\mathrm{Hz}, 3 \mathrm{H}), 1.87-1.83(\mathrm{~m}, 3 \mathrm{H}), 1.71-1.61(\mathrm{~m}, 6 \mathrm{H}) ;{ }^{13} \mathrm{C} \mathrm{NMR}\left(125 \mathrm{MHz}, \mathrm{CDCl}_{3}\right): \delta 174.0$, 148.7, 131.2, 108.7, 66.4, 66.3, 34.2, 34.0, 23.3, 22.1, 11.3; $\operatorname{IR}\left(\mathrm{cm}^{-1}\right): 3357,2923,2852,1757$, 1660, 1435; MS (APCI+ev): m/z (\%): 225 (100) $[M+H]^{+}, 207$ (20), 181 (30), 163 (55), 153 
(85), 135 (100), 107 (10); HRMS (ES) calcd. for $\mathrm{C}_{12} \mathrm{H}_{17} \mathrm{O}_{4}(\mathrm{M}+\mathrm{H})$ : 225.1127; found 225.1119 .

$7 \mathbf{f}$

${ }^{1} \mathrm{H}$ NMR $\left(300 \mathrm{MHz}, \mathrm{CDCl}_{3}\right): \delta 7.58(\mathrm{~d}, J=5.7 \mathrm{~Hz}, 1 \mathrm{H}), 6.00(\mathrm{~d}, J=6 \mathrm{~Hz}, 1 \mathrm{H}), 3.51-3.35$ (m, $4 \mathrm{H}), 2.26-2.15(\mathrm{~m}, 1 \mathrm{H}), 2.10-2.04(\mathrm{~m}, 2 \mathrm{H}), 1.89-1.65(\mathrm{~m}, 3 \mathrm{H}), 1.16(\mathrm{t}, J=6.6 \mathrm{~Hz}, 3$ $\mathrm{H}), 1.05(\mathrm{t}, J=7.2 \mathrm{~Hz}, 3 \mathrm{H}) ;{ }^{13} \mathrm{C} \mathrm{NMR}\left(75 \mathrm{MHz}, \mathrm{CDCl}_{3}\right): \delta 172.3,157.4,121.2,110.4,96.3$, 59.6, 57.0, 34.2, 31.1, 18.7, 15.7, 15.4; IR $\left(\mathrm{cm}^{-1}\right):$ 2954, 2911, 1750, 1677, 1411; MS (APCI+ev): $m / z(\%): 227$ (100) $[M+H]^{+}, 209$ (5), 181 (15); HRMS (ES) calcd. for $\mathrm{C}_{12} \mathrm{H}_{18} \mathrm{O}_{4} \mathrm{Na}(\mathrm{M}+\mathrm{Na}): 249.1103$; found 249.1102.

$7 \mathrm{~g}$

${ }^{1} \mathrm{H}$ NMR (300 MHz, $\left.\mathrm{CDCl}_{3}\right): \delta 7.16(\mathrm{~m}, 1 \mathrm{H}), 3.55-3.35$ (m, $\left.4 \mathrm{H}\right), 2.27-2.17(\mathrm{~m}, 1 \mathrm{H}), 2.12-$ $2.02(\mathrm{~m}, 2 \mathrm{H}), 1.90$ (s, $3 \mathrm{H}), 1.94-1.64(\mathrm{~m}, 3 \mathrm{H}) ;{ }^{13} \mathrm{C} \mathrm{NMR}\left(75 \mathrm{MHz}, \mathrm{CDCl}_{3}\right): \delta 173.3,149.4$, 129.6, 110.1, 93.9, 59.6, 56.9, 34.1, 30.8, 18.5, 15.7, 15.4, 10.8; IR( $\left(\mathrm{cm}^{-1}\right): 3200,2920,1755$, 1429 ; MS (APCI+ev): $m / z(\%): 241$ (15) $[M+H]^{+}, 195$ (90), 177 (25), 149 (100), 121 (5), 79 (15); Elemental analysis calcd. for $\mathrm{C}_{13} \mathrm{H}_{20} \mathrm{O}_{4} \mathrm{C}: 64.98 \%$; $\mathrm{H} 8.39 \%$; found $\mathrm{C} 65.06 \%$; $\mathrm{H}$ $8.19 \%$.

$7 \mathrm{~h}$

${ }^{1} \mathrm{H}$ NMR (300 MHz, $\mathrm{CDCl}_{3}$ ): $\delta 5.00$ (s, $\left.1 \mathrm{H}\right), 3.86$ (s, $\left.3 \mathrm{H}\right), 3.66-3.40$ (m, $\left.4 \mathrm{H}\right), 2.21-1.95$ (m, $4 \mathrm{H}), 1.89-1.61(\mathrm{~m}, 2 \mathrm{H}), 1.16-1.09$ (apparent q, $J=7.0 \mathrm{~Hz}, 6 \mathrm{H}) ;{ }^{13} \mathrm{C} \mathrm{NMR}(75 \mathrm{MHz}$, $\left.\mathrm{CDCl}_{3}\right): \delta 183.2,171.7,108.9,92.1,88.9,59.7,58.5,57.7,32.4,31.9,17.7,15.6,15.3$; IR( $\left(\mathrm{cm}^{-1}\right): 3050,2925,1750,1438$; MS (APCI+ev): $\mathrm{m} / z(\%): 257$ (10) $[M+H]^{+}, 211(50), 179$ (25), 165 (100), 133 (10); HRMS (ES) calcd. for $\mathrm{C}_{13} \mathrm{H}_{21} \mathrm{O}_{5}(\mathrm{M}+\mathrm{H})$ : 257.1389; found 257.1401 .

$7 \mathbf{i}$

${ }^{1} \mathrm{H}$ NMR (300 MHz, $\left.\mathrm{CDCl}_{3}\right): \delta 5.03$ (s, $\left.1 \mathrm{H}\right), 3.90$ (s, $\left.3 \mathrm{H}\right), 3.32$ (s, $\left.3 \mathrm{H}\right), 3.24$ (s, $\left.3 \mathrm{H}\right), 2.23-$ 1.97 (m, $4 \mathrm{H}), 1.86-1.63$ (m, $2 \mathrm{H}) ;{ }^{13} \mathrm{C} \mathrm{NMR}$ (75 MHz, $\left.\mathrm{CDCl}_{3}\right): \delta 182.9,171.4,109.3,91.8$, 89.0, 59.7, 50.9, 49.9, 32.6, 30.9, 17.5; IR( $\left.\mathrm{cm}^{-1}\right): 3116,2837,1751,1622,1456,1440$; MS (APCI+ev): $m / z(\%): 229$ (35) $[M+H]^{+}, 197$ (50), 165 (100); HRMS (ES) calcd. for $\mathrm{C}_{11} \mathrm{H}_{17} \mathrm{O}_{5}$ (M+H): 229.1076; found 229.1076. 
General procedure for the conjugate reduction of spirocyclic butenolides $7 \mathrm{~b}-7 \mathrm{c}$.

A solution of $7 \mathbf{b}-7 \mathbf{c}(1 \mathrm{mmol}, 1$ eq. $)$ in methanol $(3 \mathrm{~mL})$ was cooled to $0^{\circ} \mathrm{C}$ and treated with $\mathrm{NiCl}_{2} \cdot 6 \mathrm{H}_{2} \mathrm{O}$ (0.7 mmol, 0.7 eq.). The resulting mixture was stirred at the same temperature for $15 \mathrm{~min}$ before the addition of $\mathrm{NaBH}_{4}(1 \mathrm{mmol}, 1$ eq.). The reaction was gradually allowed to reach room temperature, after which it was quenched with a saturated solution of $\mathrm{NH}_{4} \mathrm{Cl}$. Following conventional extractive workup, the products $\mathbf{1 2 b - 1 2 c}$ were obtained in analytically pure form.

12b

${ }^{1} \mathrm{H}$ NMR (500 MHz, $\left.\mathrm{CDCl}_{3}\right): \delta$ 4.05-3.96 (m, $\left.4 \mathrm{H}\right), 2.68-2.61(\mathrm{~m}, 1 \mathrm{H}), 2.54-2.48(\mathrm{~m}, 1 \mathrm{H})$, $2.40(\mathrm{dtd}, J=11.5,4.0,3.5 \mathrm{~Hz}, 1 \mathrm{H}), 2.11-2.06$ (m, $1 \mathrm{H}), 2.02-1.96(\mathrm{~m}, 2 \mathrm{H}), 1.92-1.81$ (m, 3 $\mathrm{H}), 1.72-1.67(\mathrm{~m}, 1 \mathrm{H}) ;{ }^{13} \mathrm{C} \mathrm{NMR}\left(125 \mathrm{MHz}, \mathrm{CDCl}_{3}\right): \delta 176.8,115.6,92.4,65.8,65.2,33.8$, 31.7, 29.1, 26.0, 17.2; IR( $\left(\mathrm{cm}^{-1}\right): 2954,2887,1770,1458,1433$; MS (APCI+ev): $m / z$ (\%): 199 (100) $[M+H]^{+}, 149$ (40), 137 (50), 109 (20); HRMS (ES) calcd. for $\mathrm{C}_{10} \mathrm{H}_{15} \mathrm{O}_{4}(\mathrm{M}+\mathrm{H})$ : 199.0970; found 199.0965.

$12 \mathrm{c}$

${ }^{1} \mathrm{H}$ NMR (300 MHz, $\left.\mathrm{CDCl}_{3}\right):$ 8 4.02-3.94 (m, $\left.4 \mathrm{H}\right), 2.74-2.61(\mathrm{~m}, 1 \mathrm{H}), 2.53-2.34(\mathrm{~m}, 2 \mathrm{H})$, 2.04-1.96 (m, $1 \mathrm{H}), 1.88-1.54(\mathrm{~m}, 8 \mathrm{H}) ;{ }^{13} \mathrm{C} \mathrm{NMR}\left(125 \mathrm{MHz}, \mathrm{CDCl}_{3}\right): \delta$ 177.1, 109.8, 88.6, 65.7, 36.0, 32.2, 29.0, 28.4, 22.6, 21.5; IR( $\left.\mathrm{cm}^{-1}\right): 2950,1771,1460 ;$ MS (APCI+ev): $\mathrm{m} / z$ (\%): 213 (100) $[M+H]^{+}$, 151 (10), 123 (5); HRMS (ES) calcd. for $\mathrm{C}_{11} \mathrm{H}_{17} \mathrm{O}_{4}(\mathrm{M}+\mathrm{H}): 213.1127$; found 213.1129.

\section{General procedure for dihydroxylation of spirocyclic butenolides $7 \mathrm{~b}-7 \mathrm{c}$.}

To a vigorously stirred solution of $\mathbf{7 b}-\mathbf{7} \mathbf{c}(0.2 \mathrm{mmol}, 1$ eq. $)$ in $\mathrm{CH}_{3} \mathrm{CN} / \mathrm{AcEt}(1 \mathrm{~mL}$ each $)$ at $0^{\circ}$ $\mathrm{C}$ was added a solution of $\mathrm{RuCl}_{3} .3 \mathrm{H}_{2} \mathrm{O}(0.014 \mathrm{mmol}, 0.07$ eq. $)$ and $\mathrm{NaIO}_{4}(0.3 \mathrm{mmol}, 1.5$ eq. $)$ in distilled water $(1 \mathrm{~mL})$. The mixture was stirred for 90 seconds after which a saturated solution of $\mathrm{Na}_{2} \mathrm{~S}_{2} \mathrm{O}_{3}$ is added. Following extractive workup with AcOEt, the dihydroxylated lactones $\mathbf{1 3 b - 1 3 c}$ are obtained in analytically pure form.

13b

${ }^{1} \mathrm{H}$ NMR $\left(500 \mathrm{MHz}, \mathrm{CDCl}_{3}\right): \delta 4.62(\mathrm{~d}, J=5.2 \mathrm{~Hz}, 1 \mathrm{H}), 4.45(\mathrm{~d}, J=5.3 \mathrm{~Hz}, 1 \mathrm{H}), 3.98-3.86$ (m, 4 H), 2.98 (br s, 1H), 2.68 (br s, $1 \mathrm{H}), 2.31-2.25$ (m, 1H), 2.08-2.01 (m, $1 \mathrm{H}), 1.99-1.95$ 
$(\mathrm{m}, 1 \mathrm{H}), 1.90-1.85(\mathrm{~m}, 2 \mathrm{H}), 1.78-1.70(\mathrm{~m}, 1 \mathrm{H}) ;{ }^{13} \mathrm{C} \mathrm{NMR}\left(125 \mathrm{MHz}, \mathrm{CDCl}_{3}\right): \delta$ 176.0, 115.7, 94.9, 70.3, 69.7, 65.9, 65.5, 32.9, 29.3, 17.6; IR( $\left.\mathrm{cm}^{-1}\right): 3367$ (br), 2924, 1772, 1132; MS (APCI+ev): $m / z(\%): 231(100)[M+H]^{+}, 213$ (10), 169 (15), 149 (5); HRMS (ES) calcd. for $\mathrm{C}_{10} \mathrm{H}_{15} \mathrm{O}_{6}(\mathrm{M}+\mathrm{H}): 231.0869$; found 231.0871.

$13 c$

${ }^{1} \mathrm{H}$ NMR $\left(300 \mathrm{MHz}, \mathrm{CDCl}_{3}\right): \delta 4.80(\mathrm{~d}, J=5.9 \mathrm{~Hz}, 1 \mathrm{H}), 4.40(\mathrm{~d}, J=5.9 \mathrm{~Hz}, 1 \mathrm{H}), 3.98-3.94$ (m, $4 \mathrm{H}), 3.12$ (br s, 1H), 2.83 (br s, $1 \mathrm{H}), 1.79-1.50$ (m, $8 \mathrm{H}) ;{ }^{13} \mathrm{C} \mathrm{NMR}\left(75 \mathrm{MHz}, \mathrm{CDCl}_{3}\right): \delta$ 176.1, 109.1, 80.8, 69.3, 69.0, 65.4, 65.2, 32.4, 29.4, 22.5, 21.1; IR(cm $\left.{ }^{-1}\right): 3354$ (br), 2911, 1770, 1412; MS (APCI+ev): $m / z$ (\%): 245 (100) $[M+H]^{+}, 201$ (15), 183 (5); HRMS (ES) calcd. for $\mathrm{C}_{11} \mathrm{H}_{17} \mathrm{O}_{6}(\mathrm{M}+\mathrm{H})$ : 245.1025; found 245.1022 . 


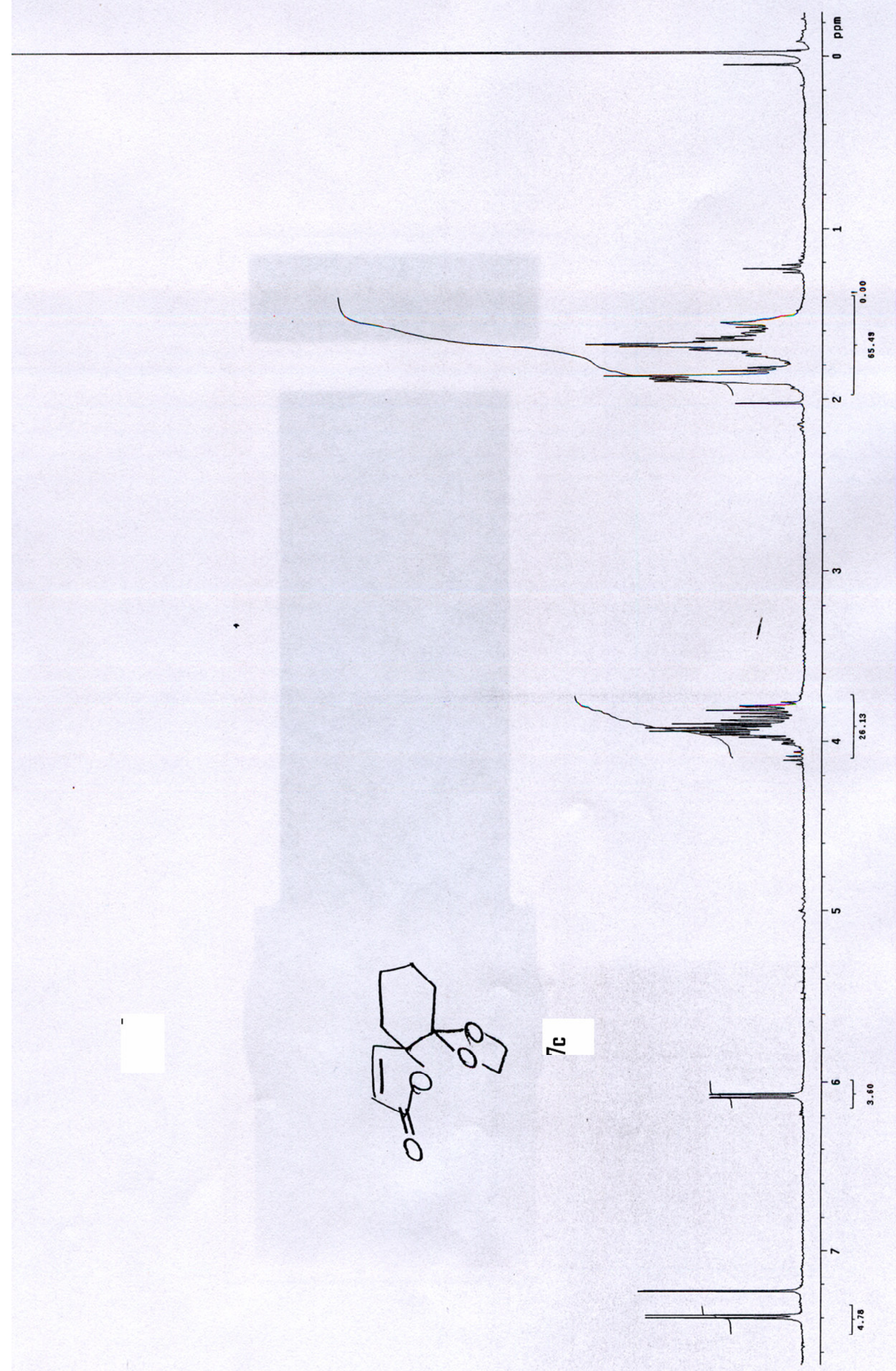




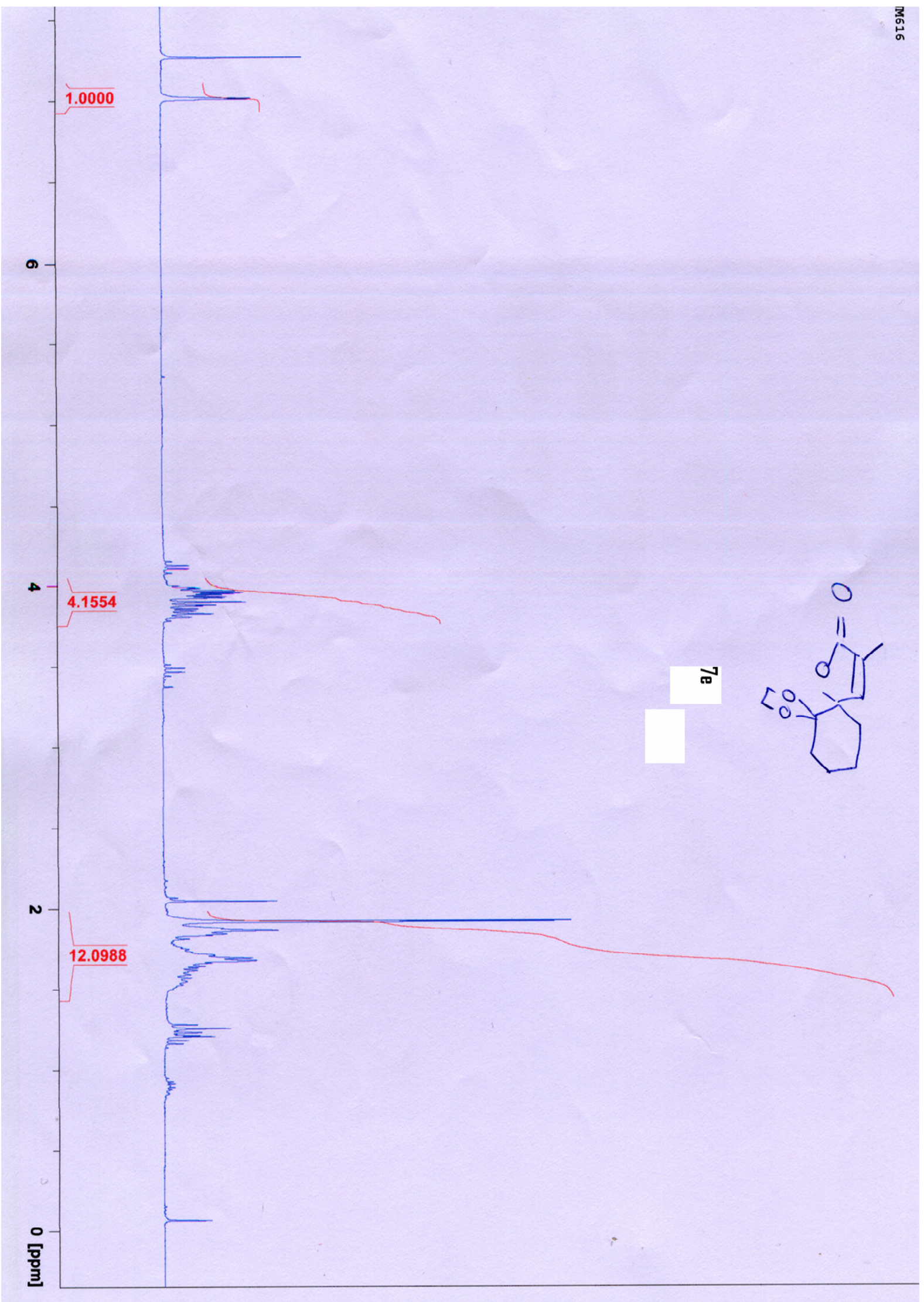




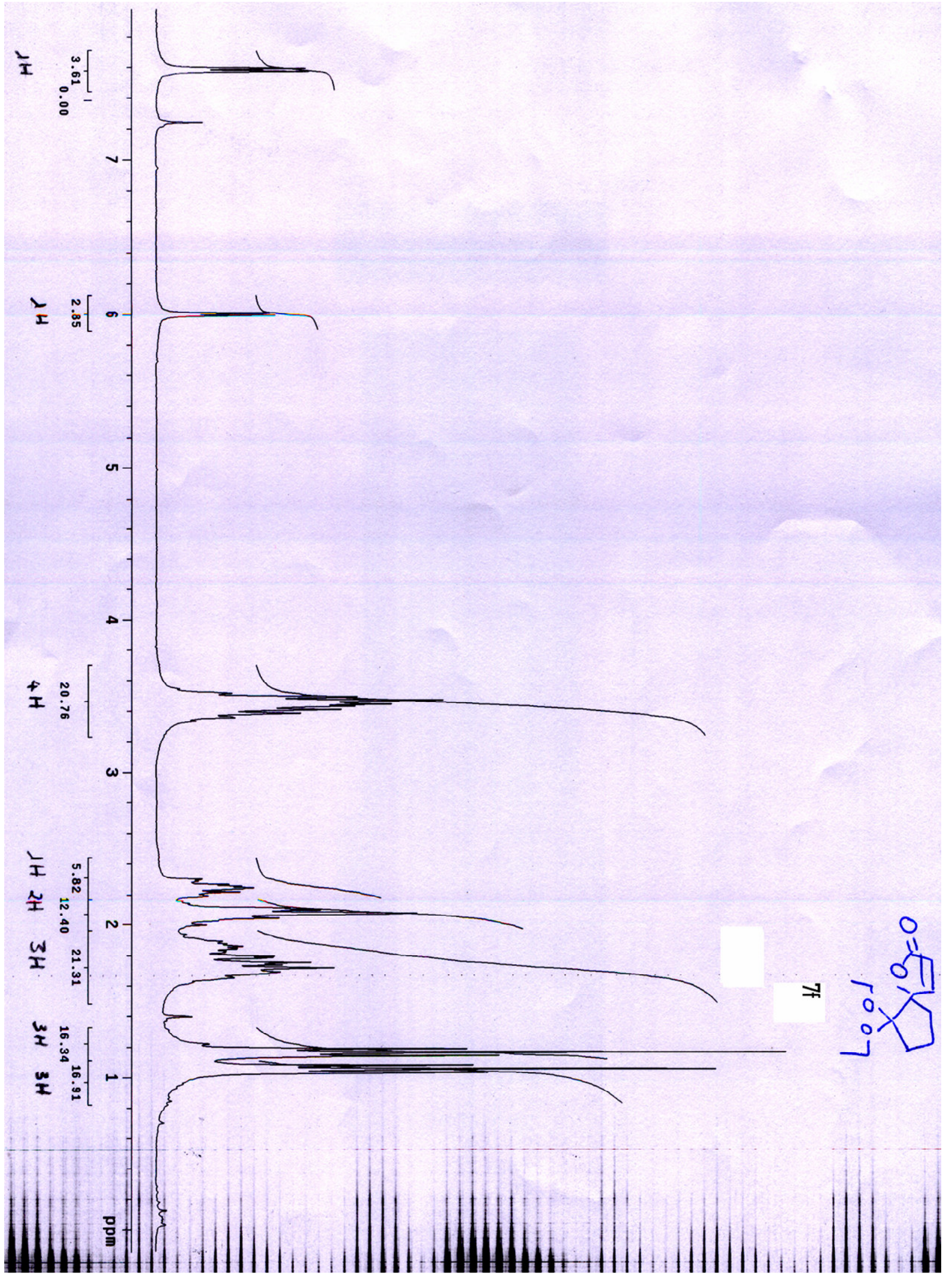




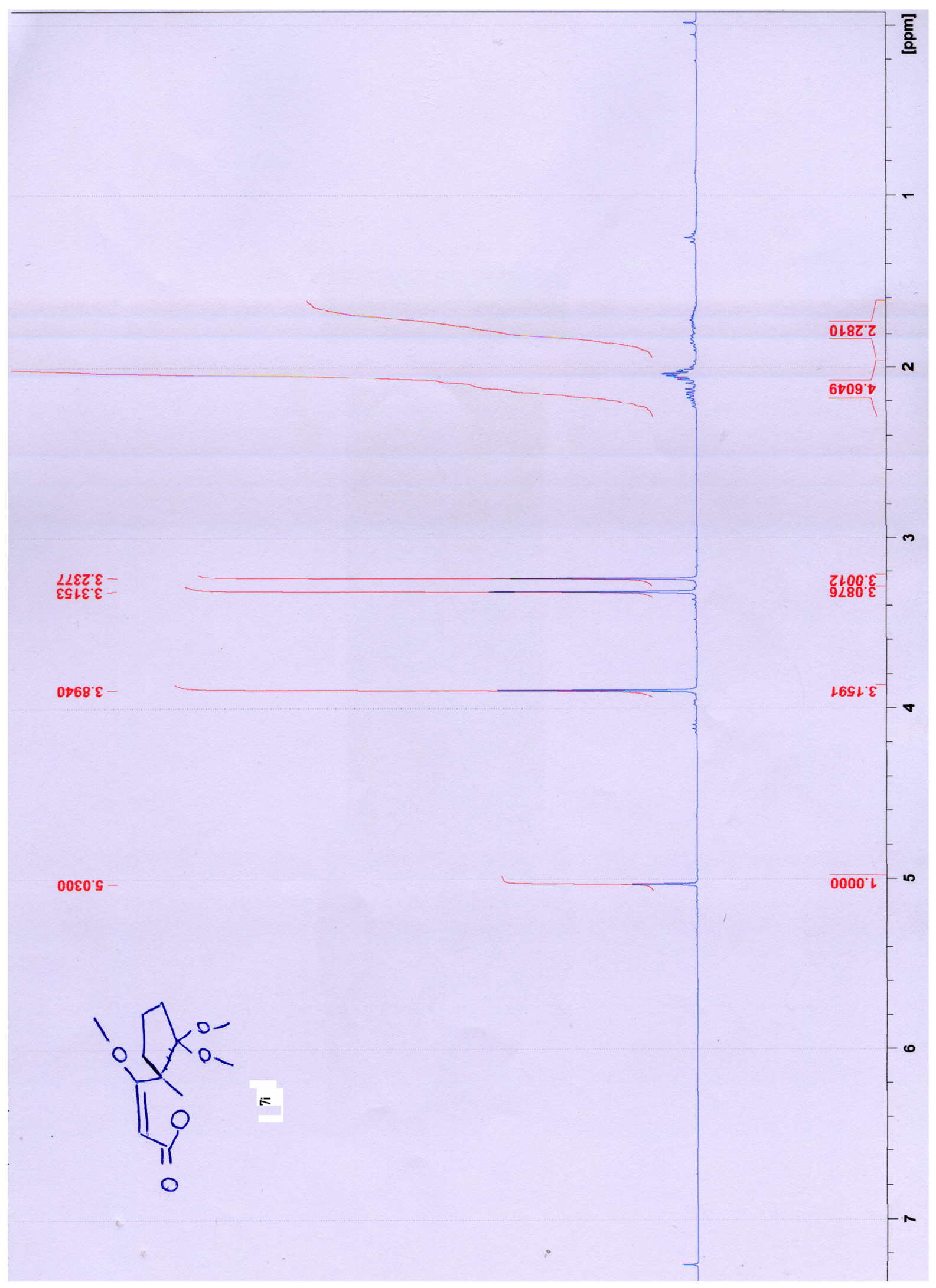




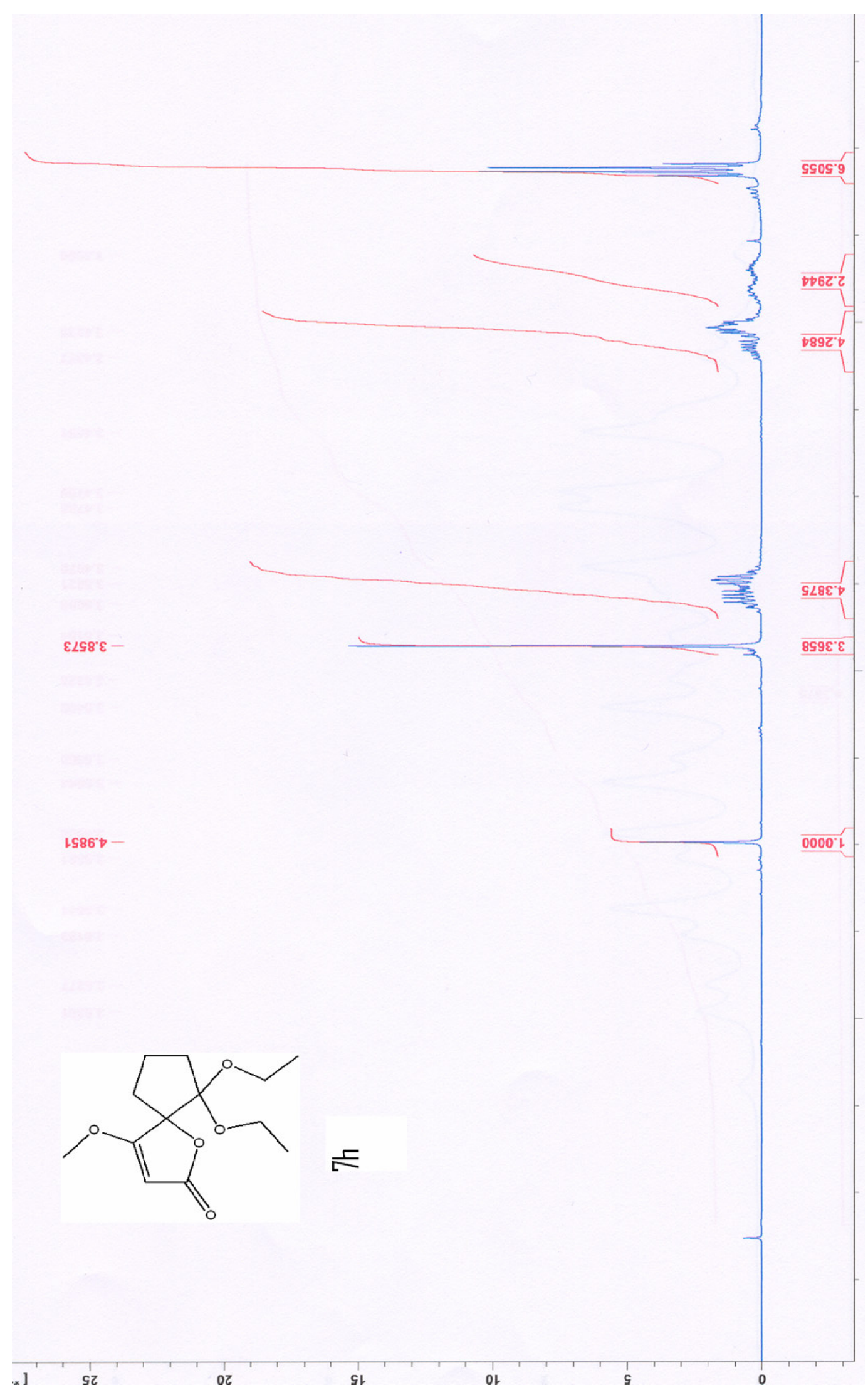




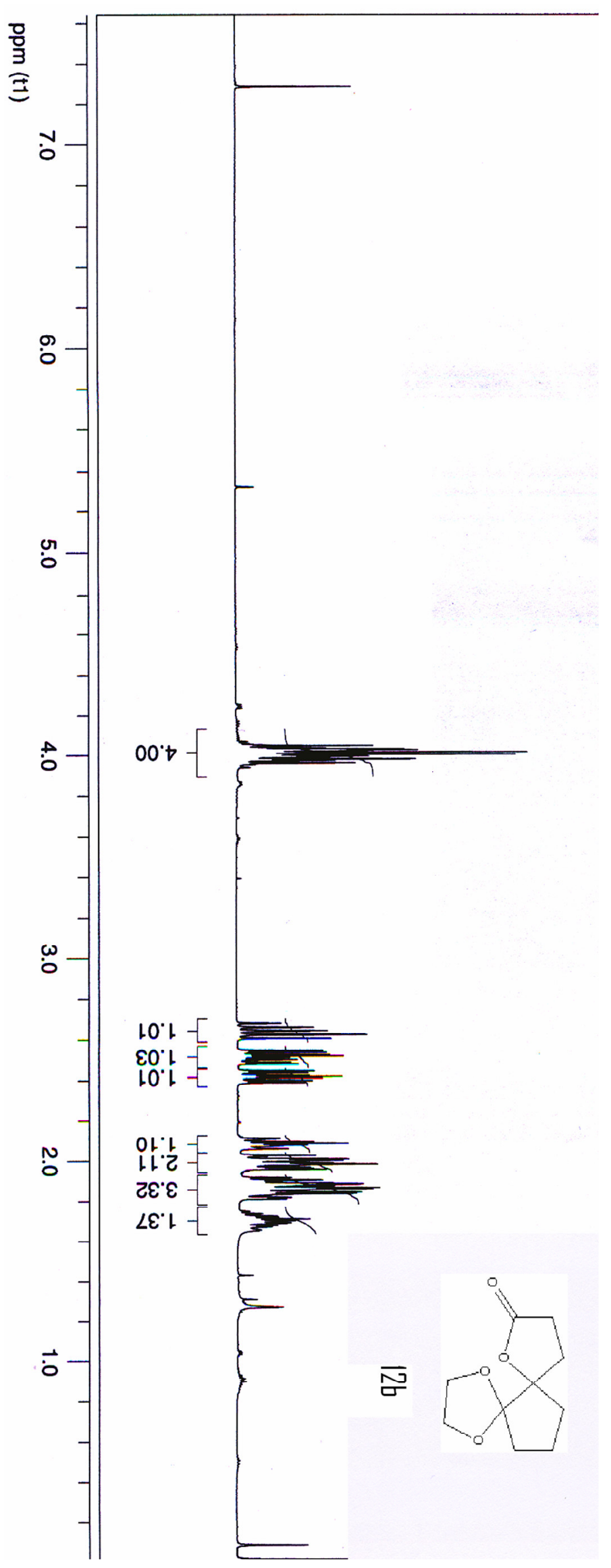




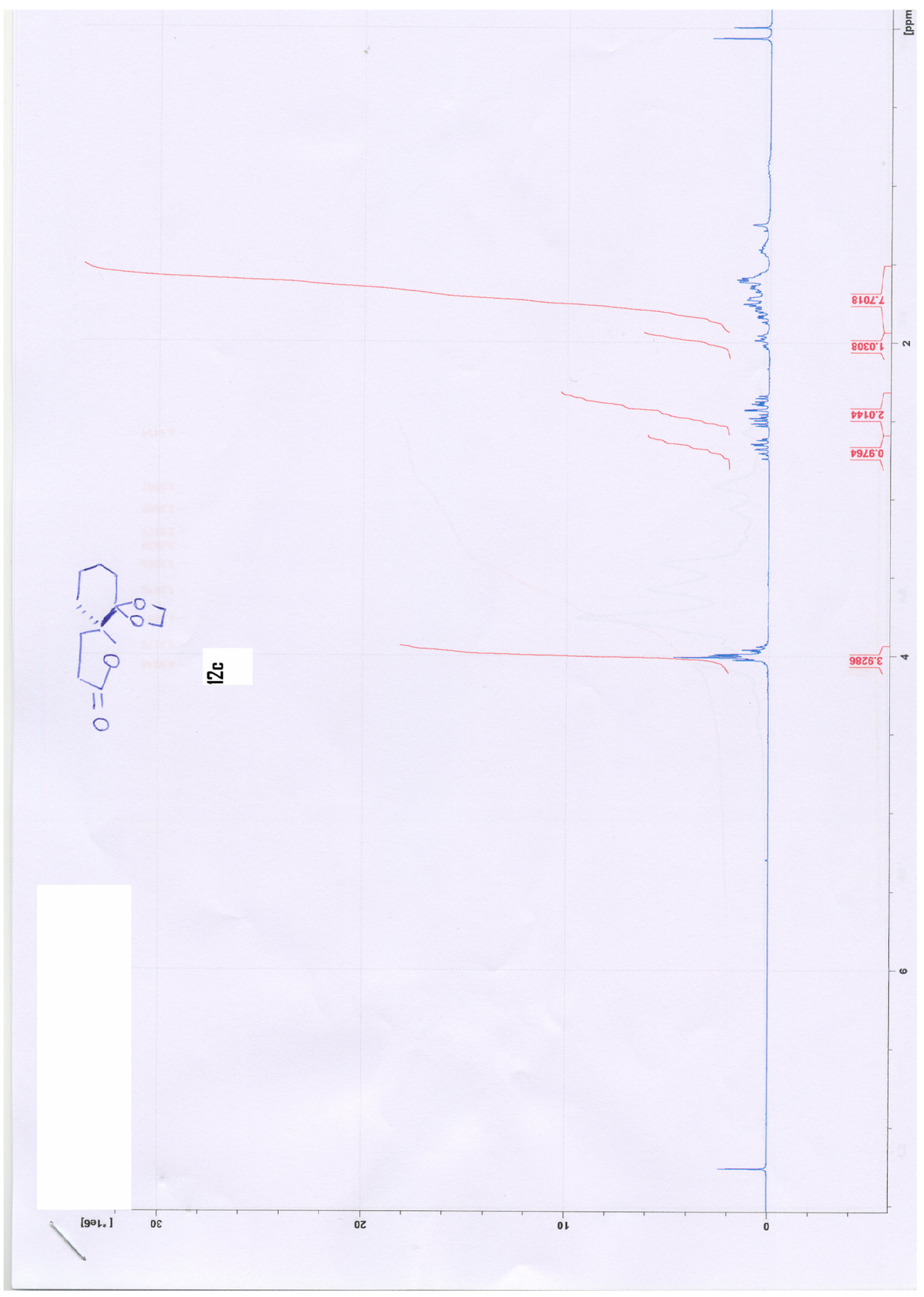




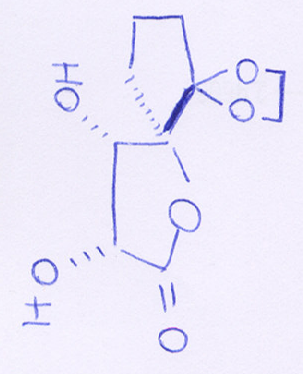

ำ

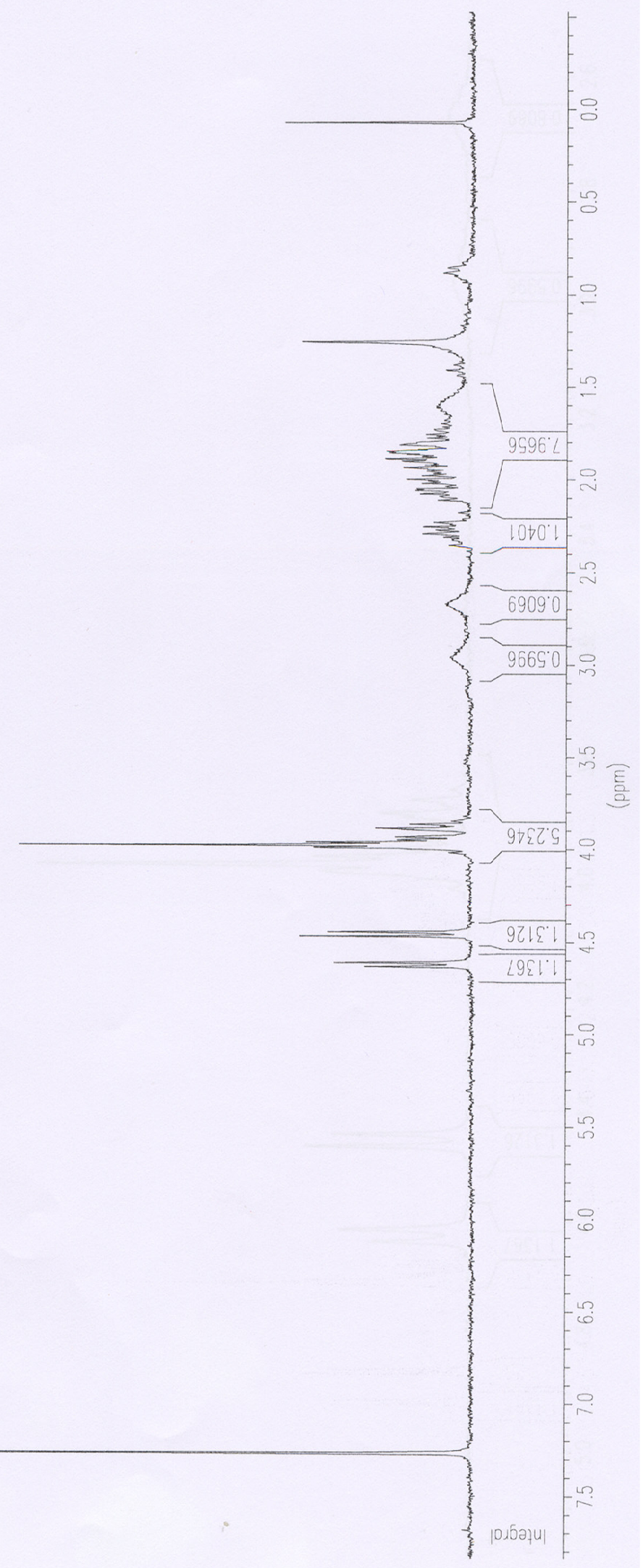




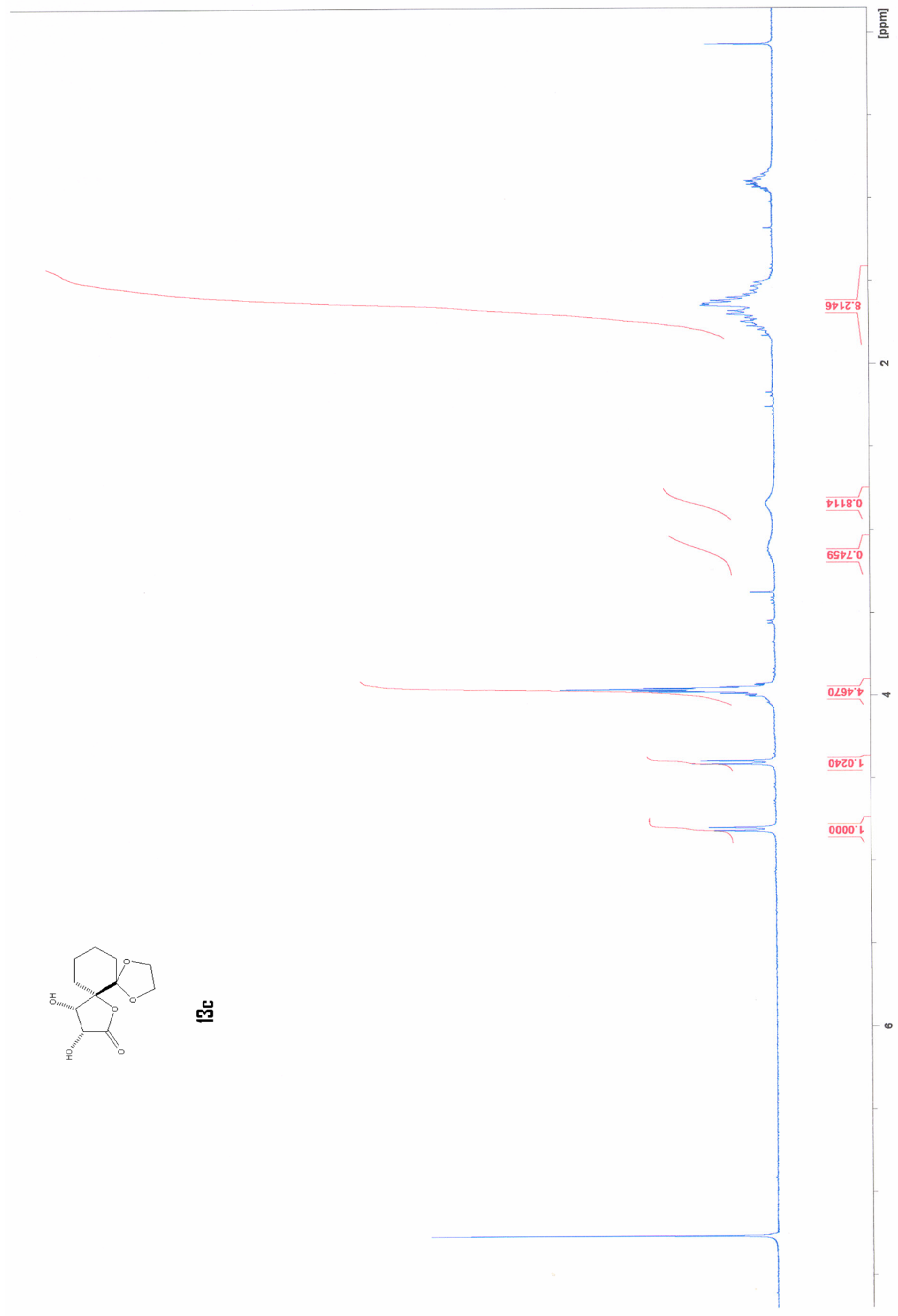

